\title{
Approximation of the Telegrapher's equations
}

\author{
G. Golo, V. Talasila, A. J. van der Schaft \\ Cornelis J. Drebbel Institute for Mechatronics, \\ Faculty of Mathematical Sciences, University of Twente \\ P.O. Box 217, 7500 AE Enschede, The Netherlands \\ t.viswanath, g.golo, a.j.vanderschaft@math.utwente.nl
}

\begin{abstract}
The problem of approximating a distributed parameter system with free boundary conditions is solved for the 1 dimensional Telegrapher's equations. The Telegrapher's equations are described using an infinite-dimensional portHamiltonian model, and we derive a finite dimensional portHamiltonian model using a mixed finite-element procedure. We show that energy conservation, passivity and some dynamic invariants are preserved in the discretization.
\end{abstract}

\section{Introduction}

In previous work, $[1,2,3]$, it has been shown how portbased network modeling of complex lumped-parameter physical systems naturally leads to a generalized Hamiltonian formulation of the dynamics. Here the geometric structure, defining together with the Hamiltonian the dynamics of the system, is given by the power-conserving interconnection structure of the system (corresponding to what in bond-graph terminology is called the "generalized junction structure"), and is called a Dirac structure. The resulting class of open dynamical systems has been called "port-Hamiltonian systems" ([3]).

Recently, the framework of port-Hamiltonian systems has been extended to classes of distributed-parameter systems [4], like Maxwell's equations over a bounded domain with energy radiation through its boundary, the $n$-dimensional wave equation, and compressible ideal fluids. Hereto a special type of infinite-dimensional Dirac structure has been introduced, based on Stokes theorem. Physically, this StokesDirac structure [4] captures the basic balance laws of the system, like Faradays and Ampères law, or mass balance. The port-Hamiltonian formulation is a non-trivial extension of the Hamiltonian formulation of PDEs by means of Poisson structures, as has been explored before in the literature ([5]). Indeed, in this case it is crucially assumed that the boundary conditions are such that the energy-flow through the boundary of the spatial domain is zero. In order to allow a non-zero boundary energy-flow the use of Dirac structures instead of Poisson structures seems to be indispensable.

As a result complex physical systems consisting of com- ponents which are either lumped-parameter or distributedparameter systems, and which moreover may belong to different physical domains can be modeled in a unified and intrinsic way. This opens up several possibilities for the analysis and control of such complex systems. From a simulation point of view, the problem concerns the incorporation of the powerful numerical methods for the solution of PDEs, such as finite-element and finite-difference methods, into this framework. Also for control purposes it is often crucial to be able to approximate either the distributedparameter system with a finite-dimensional system, or to approximate the infinite-dimensional controller by a finitedimensional one. This is however not an easy task. One fundamental problem which arises is the fact that numerical methods for the solution of PDEs usually assume that the boundary conditions are given. On the other hand, more often than not it is precisely the boundary conditions which represent the interaction of the distributed-parameter component with the other components of the system. We thus have to approximate the distributed-parameter system while retaining the energetic port-structure of the system. In this paper we show how this can be done for the example of the transmission line. Indeed, we will show how the intrinsic Hamiltonian formulation of the transmission line suggests finite-element methods which result in finite-dimensional systems which are again port-Hamiltonian! From a geometric point of view it is natural to use different finite-elements for the approximation of the differential forms (which are the physical variables of the system), see e.g. [6] where this is shown to lead to mixed finite elements. In the present paper we have to deal with the additional complication of the boundary variables, which we in fact solve in the same spirit as of [6]. Finally, we show how the proposed method preserves some physical properties of interest, like energy conservation, passivity, some dynamic invariants etc, in the finite-model also.

\section{The Telegrapher's equations}

We recall the definition of a Dirac structure from [4]. Let $\mathcal{F}$ and $\mathcal{E}$ be linear spaces (usually called the spaces of flows and efforts), equipped with a non-degenerate pairing that is 
a bilinear operation

$$
\mathcal{F} \times \mathcal{E} \rightarrow L
$$

with $L$ a linear space. The pairing will be denoted by $\langle e \mid f\rangle \in L, f \in \mathcal{F}, e \in \mathcal{E}$. Flows (or rate energy variables) are denoted by $f$, and efforts (or co-energy variables) are denoted by $e$. By symmetrizing the pairing (see [4]) we obtain a symmetric bilinear form $\langle\langle\rangle$,$\rangle on \mathcal{F} \times \mathcal{E}$.

Definition 1 Let $\mathcal{F}$ and $\mathcal{E}$ be linear spaces with a nondegenerate pairing $\langle\mid\rangle$. A Dirac structure is a linear subspace $\mathcal{D} \subset \mathcal{F} \times \mathcal{E}$ such that $\mathcal{D}=\mathcal{D}^{\perp}$, with $\perp$ denoting the orthogonal complement with respect to the symmetric bilinear form $\langle\langle\rangle$,$\rangle .$

In the finite-dimensional case the above definition of a Dirac structure reduces to a subspace $\mathcal{D} \subset \mathcal{F} \times \mathcal{E}$ with the property that $\langle e \mid f\rangle=0, \forall(f, e) \in \mathcal{D}$, and that $\operatorname{dim} \mathcal{D}=\operatorname{dim} \mathcal{E}=\operatorname{dim} \mathcal{F}$.

Next, we recall the port-Hamiltonian model of the transmission line [4]. Consider an ideal lossless transmission line with $Z=[0,1] \subset \mathbb{R}$. We define the energy variables as the infinitesimal charge $q=q(t, z) \in \Omega^{1}(Z)$, and the infinitesimal fiux $\phi=\phi(t, z) \in \Omega^{1}(Z)$, where $\Omega^{1}(Z)$ denotes the space of 1 -forms. The energy density (or the Hamiltonian density) stored at time $t$ in the transmission line is given as:

$$
\mathcal{H}(q, \phi)=\frac{1}{2}\left(q(t, z) \wedge * \frac{q(t, z)}{C(z)}+\phi(t, z) \wedge * \frac{\phi(t, z)}{L(z)}\right)
$$

where $*$ is the Hodge star operator which is usually defined as $*(U): \Omega^{k}(U) \rightarrow \Omega^{n-k}(U)$ where $U$ is open in $Z$, and $Z$ is a Riemannian manifold. Of course the total energy is then $H=\int_{Z} \mathcal{H}$. The co-energy variables (voltage and current) are related to the energy variables by the constitutive relations as follows:

$$
\begin{aligned}
& e^{q}(t, z)=\frac{\partial \mathcal{H}}{\partial q}=* \frac{1}{C(z)} q(t, z) \in \Omega^{0}(Z) \quad(\text { voltage }) \\
& e^{\phi}(t, z)=\frac{\partial \mathcal{H}}{\partial \phi}=* \frac{1}{L(z)} \phi(t, z) \in \Omega^{0}(Z) \quad \text { (current) }
\end{aligned}
$$

Next we denote the rate energy variables as follows:

$$
f^{q}(t, z)=\frac{\partial q(t, z)}{\partial t}, \quad f^{\phi}(t, z)=\frac{\partial \phi(t, z)}{\partial t}
$$

The resulting port-Hamiltonian system (with the boundary conditions) is given by the Telegrapher's equations:

$$
\begin{aligned}
& {\left[\begin{array}{l}
f^{q}(t, z) \\
f^{\phi}(t, z)
\end{array}\right]=\left[\begin{array}{cc}
0 & -d \\
-d & 0
\end{array}\right]\left[\begin{array}{l}
e^{q}(t, z) \\
e^{\phi}(t, z)
\end{array}\right]} \\
& {\left[\begin{array}{l}
e^{B L} \\
e^{B R} \\
f^{B L} \\
f^{B R}
\end{array}\right]=\left[\begin{array}{llll}
1 & 0 & 0 & 0 \\
0 & 1 & 0 & 0 \\
0 & 0 & 1 & 0 \\
0 & 0 & 0 & 1
\end{array}\right]\left[\begin{array}{l}
e^{q}(t, 0) \\
e^{q}(t, 1) \\
e^{\phi}(t, 0) \\
e^{\phi}(t, 1)
\end{array}\right]}
\end{aligned}
$$

where $d$ is the usual exterior-derivative ${ }^{1}$. $B$ denotes the boundary and $B L, B R$, denote the left and right boundary respectively. It has been shown in [4] that (3) defines the so-called Stokes-Dirac structure, and (2),(3) together define a port-Hamiltonian system.

\subsection{Conservation laws}

In this subsection we present some conservation laws of the port-Hamiltonian system (3). First, we express the energy conservation law of this system:

$\frac{d H(q(t), \phi(t))}{d t}=\int_{\partial Z} e^{B}(t) \wedge f^{B}(t)=\left[f^{B L}(t) e^{B L}(t)-f^{B R}(t) e^{B R}(t)\right]$

which expresses that the increase in energy inside the domain $Z$ is equal to the supplied energy through the boundary $\partial Z$. From this we derive the passivity (see [3]) property of this system as follows: integrating (4) on both sides with respect to time, we obtain

$$
\begin{aligned}
& H(q(T), \phi(T))-H(q(0), \phi(0))=\int_{0}^{T} \int_{\partial z} e^{B}(t) \wedge f^{B}(t) \\
& \Rightarrow \int_{0}^{T} s\left(e^{B}(t), f^{B}(t)\right) \geq-H(q(0), \phi(0))
\end{aligned}
$$

where $\int_{0}^{T} s\left(e^{B}(t), f^{B}(t)\right)=\int_{0}^{T} \int_{\partial z} e^{B}(t) \wedge f^{B}(t)$ is the supplied power. (5) implies that the input-output maps of the system for every $(q(0), \phi(0))$ are passive.

We can obtain two dynamic invariants by integrating the first part of (3) over $Z$. First we obtain:

$$
\dot{Q}(t)=e^{\phi}(t, 0)-e^{\phi}(t, 1)=f^{B L}(t)-f^{B R}(t)
$$

where $Q(t)=\int_{Z} q(t, z)$ is the total charge of the transmission line. Next, we obtain:

$$
\dot{\Phi}(t)=e^{q}(t, 0)-e^{q}(t, 1)=e^{B L}(t)-e^{B R}(t)
$$

where $\Phi(t)=\int_{Z} \phi(t, z)$ is the total flux of the transmission line. Equation (6) represents charge conservation, and (7) represents flux conservation, see [4].

\section{Spatial Discretization of Telegrapher's Equations}

We present a discretization procedure for obtaining a finitedimensional model of the Telegrapher's Equations which preserves certain physical properties of interest (see Section 3.1). The procedure consists of two steps: first we discretize the interconnection structure in (3), and then discretize the constitutive relations (2) of the energy storage part of the transmission line.

\footnotetext{
${ }^{1} d$ is defined as $d(U): \Omega^{k}(U) \rightarrow \Omega^{k+1}(U)$ where $U$ is open in $Z$. In other words the exterior derivative maps a space of $k$-forms to a space of $k-1$ forms
} 


\subsection{Discretization of the interconnection structure}

The transmission line is 'split' into $n$ cells. Due to the spatial compositionality (i.e. interconnection of two transmission lines via a common boundary once again gives a transmission line) property, we need to perform the discretization to only one cell. For ease of notation we concentrate on the first cell, we denote the spatial manifold of the first cell as $Z=[0, S], S$ being the length of this cell.

The relations between the boundary variables and the efforts (or co-energy variables) of this cell are

$$
\begin{array}{ll}
e^{B L}(t)=e^{q}(t, 0), & e^{B R}(t)=e^{q}(t, S) \\
f^{B L}(t)=e^{\phi}(t, 0), & f^{B R}(t)=e^{\phi}(t, S)
\end{array}
$$

The infinitesimal charge rate $f^{q}(t, z)$ and the infinitesimal flux rate $f^{\phi}(t, z)$ are approximated on $Z$ as follows:

$$
f^{q}(t, z)=f^{q}(t) \cdot{ }^{1} b^{q}(z), \quad f^{\phi}(t, z)=f^{\phi}(t) \cdot{ }^{1} b^{\phi}(z)
$$

where we assume that the approximating one-forms ${ }^{1} b^{q},{ }^{1} b^{\phi} \in \Omega^{1}(Z)$ satisfy the following conditions

$$
\int_{z}^{1} b^{q}(z)=1, \quad \int_{z}^{1} b^{\phi}(z)=1
$$

The above assumption is physically motivated because as we see later on, the infinitesimal charge and the infinitesimal flux will be approximated on $z$ as $q(t, z)=Q(t) \cdot \cdot^{1} b^{q}(z)$, and $\phi(t, z)=\Phi(t) .{ }^{1} b^{\phi}(z)$ so that by $(10) Q(t)$ and $\Phi(t)$ represent the total charge and total fux of this cell. The efforts $e^{q}(t, z)$ and $e^{\phi}(t, z)$ are approximated as

$$
\begin{aligned}
& e^{q}(t, z)=e_{0}^{q}(t) \cdot b_{0}^{q}(z)+e_{S}^{q}(t) \cdot b_{S}^{q}(z) \\
& e^{\phi}(t, z)=e_{0}^{\phi}(t) \cdot b_{0}^{\phi}(z)+e_{S}^{\phi}(t) b_{S}^{\phi}(z)
\end{aligned}
$$

where we assume that the approximating 0 -form elements $b_{0}^{q}, b_{S}^{q}, b_{0}^{\phi}, b_{S}^{\phi} \in \Omega^{0}(z)$ satisfy the following conditions

$$
\begin{aligned}
& b_{0}^{q}(0)=1, \quad b_{0}^{q}(S)=0, \quad b_{S}^{q}(0)=0, \quad b_{S}^{q}(S)=1 \\
& b_{0}^{\phi}(0)=1, \quad b_{0}^{\phi}(S)=0, \quad b_{S}^{\phi}(0)=0, \quad b_{S}^{\phi}(S)=1
\end{aligned}
$$

The above assumption is made since then $e^{q}(t, z)$, respectively $e^{\phi}(t, z)$, coincides with $e_{0}^{q}(t)$, respectively $e_{0}^{\phi}(t)$, at the point 0 , and with $e_{S}^{q}(t)$, respectively $e_{S}^{\phi}(t)$, at the point $S$. Also, the efforts cannot be approximated with only one approximating element, see [7].

By substituting (11) and (9) into (3) we obtain

$$
f^{q}(t) \cdot{ }^{\prime} b^{q}(z)=-d b_{0}^{\phi}(z) \cdot e_{0}^{\phi}(t)-d b_{S}^{\phi}(z) \cdot e_{S}^{\phi}(t)
$$

The forms ${ }^{1} b^{q}(z), b_{0}^{\phi}(z), b_{S}^{\phi}(z)$ should be chosen in such a way that for every $e_{0}^{\phi}(t), e_{S}^{\phi}(t)$ we can find a $f^{q}(t)$ such that (13) is satisfied. Using (10) and (12) we obtain

$$
b_{0}^{\phi}(z)=1-\int_{0}^{z} b^{q}(p), \quad b_{S}^{\phi}(z)=\int_{0}^{z} b^{q}(p)
$$

Similarly we can obtain as before

$$
b_{0}^{q}(z)=1-\int_{0}^{z} b^{\phi}(p), \quad b_{S}^{q}(z)=\int_{0}^{z} b^{\phi}(p)
$$

By substituting (14) into (13) we obtain

$$
f^{q}(t) \cdot{ }^{1} b^{q}(z)=e_{0}^{\phi}(t) \cdot{ }^{1} b^{q}(z)-e_{S}^{\phi}(t) \cdot{ }^{1} b^{q}(z)
$$

Integrating the above equation over $z$, we have

$$
f^{q}(t)=e_{0}^{\phi}(t)-e_{S}^{\phi}(t)
$$

Similarly, we can show that

$$
f^{\phi}(t)=e_{0}^{q}(t)-e_{S}^{q}(t)
$$

Thus, the relations describing the spatially discretized interconnection structure of this particular cell are given by

$$
\left[\begin{array}{c}
e^{\mathrm{BL}}(t) \\
e^{\mathrm{BR}}(t) \\
f^{\mathrm{BL}}(t) \\
f^{\mathrm{BR}}(t) \\
f^{q}(t) \\
f^{\phi}(t)
\end{array}\right]=\left[\begin{array}{cccc}
1 & 0 & 0 & 0 \\
0 & 1 & 0 & 0 \\
0 & 0 & 1 & 0 \\
0 & 0 & 0 & 1 \\
0 & 0 & 1 & -1 \\
1 & -1 & 0 & 0
\end{array}\right]\left[\begin{array}{l}
e_{0}^{q}(t) \\
e_{S}^{q}(t) \\
e_{0}^{\phi}(t) \\
e_{S}^{\phi}(t)
\end{array}\right]
$$

Now, we describe some properties of the approximating 0 forms and 1-forms

Proposition $1 b^{q}(z), b^{\phi}(z),{ }^{1} b^{q}(z),{ }^{1} b^{\phi}(z)$ satisfy the following conditions:

(i) $b_{0}^{q}(z)+b_{S}^{q}(z)=1$

(ii) $b_{0}^{\phi}(z)+b_{S}^{\phi}(z)=1$

(iii) $\int_{z} b_{0}^{q}(z) \wedge^{1} b^{q}(z)+\int_{z} b_{S}^{q}(z) \wedge^{1} b^{q}(z)=1$

(iv) $\int_{z} b_{0}^{\phi}(z) \wedge^{1} b^{\phi}(z)+\int_{z} b_{S}^{\phi}(z) \wedge^{1} b^{\phi}(z)=1$

(v) $\int_{z} b_{0}^{q}(z) \wedge^{1} b^{q}(z)=\int_{z} b_{S}^{\phi}(z) \wedge^{1} b^{\phi}(z)$

Proof: (i) and (ii) are trivial to prove and the proof of (iv) follows from the proof of (iii). We prove only (iii) and (v).

(iii) $\int_{z} b_{0}^{q}(z) \wedge^{1} b^{q}(z)+\int_{z} b_{S}^{q}(z) \wedge^{1} b_{i}^{q}(z)$ $=\int_{z}\left(b_{0}^{q}(z)+b_{S}^{q}(z)\right) \wedge^{\prime} b^{q}(z)=1$

(v) $\int_{z} b_{0}^{q}(z) \wedge^{1} b^{q}(z)=\int_{z}{ }^{1} b^{q}(z) \wedge\left(1-\int_{0}^{z}{ }^{1} b^{\phi}(p)\right)$

$$
\begin{aligned}
& =\left.\left[\left(1-\int_{0}^{z 1} b^{\phi}(p)\right) \cdot \int_{0}^{z 1} b^{q}(p)\right]\right|_{0} ^{S}+ \\
& \int_{z}{ }^{1} b^{\phi}(z) \wedge \int_{0}^{z} b^{q}(p)=\int_{z} b_{S}^{\phi}(z) \wedge^{1} b^{\phi}(z) .
\end{aligned}
$$

The net power of this cell is

$$
P^{n e t}=\int_{z} e^{\phi}(z) \wedge f^{\phi}(z)+\int_{z} e^{q}(z) \wedge f^{q}(z)-e^{B L} f^{B L}+e^{B R} f^{B R}
$$


Inserting the corresponding terms from (9) and (11) and using the properties of Proposition 1, we have

$$
\begin{aligned}
p^{\text {net }}= & \left(\alpha e_{0}^{q}+(1-\alpha) e_{S}^{q}\right) f^{q}+ \\
& \left((1-\alpha) e_{0}^{\phi}+\alpha e_{S}^{\phi}\right) f^{\phi}-e_{0}^{\mathrm{BL}} f^{\mathrm{BL}}+e_{0}^{\mathrm{BR}} f^{\mathrm{BR}}
\end{aligned}
$$

where

$$
\alpha=\int_{z} b^{q}(z) \wedge^{1} b^{q}(z)
$$

The expression (20a) is used for the identification of the port variables of the spatially discretised interconnection structure of this cell. The third and the fourth term on the right side of $(20 \mathrm{a})$ imply that the port variables of the left boundary port are $\left(f^{\mathrm{BL}}, e^{\mathrm{BL}}\right)$ and that the port variables of the right boundary port are $\left(f^{\mathrm{BR}}, e^{\mathrm{BR}}\right)$. The first term on the right side of $(20 \mathrm{a})$, denotes the power supplied to or taken from the electrical part of this cell, and that the flow variable of the electric port is $f^{q}$ and that the effort variable is $\alpha e_{0}^{q}+(1-\alpha) e_{S}^{q}$. Similarly, the second term implies that the port variables of the magnetic port are $\left(f^{\phi}, \alpha e_{0}^{\phi}+(1-\alpha) e_{S}^{\phi}\right)$. If we introduce the following replacements

$$
\begin{gathered}
\bar{f}^{q}=f^{q}, \\
\bar{f}^{\phi}=f^{\phi}, \\
\bar{e}^{q}=\alpha e_{0}^{q}+(1-\alpha) e_{S}^{q}, \\
\bar{e}^{\phi}=(1-\alpha) e_{0}^{\phi}+\alpha e_{S}^{\phi} .
\end{gathered}
$$

then the expression for $P^{\text {nut }}$ becomes

$$
P^{n e t}=\langle\mathbf{e} \mid \mathbf{f}\rangle=\bar{f}^{q} \bar{e}^{q}+\bar{f}^{\phi} \bar{e}^{\phi}-e^{B L} f^{B L}+e^{B R} f^{B R},
$$

where $\mathbf{f}^{T}=\left[\bar{f}^{q} \bar{f}^{\phi} f^{B L} f^{B R}\right]$ and $\mathbf{e}^{T}=\left[\bar{e}^{q} \bar{e}^{\phi} e^{B L} e^{B R}\right]$.

Remark 1 (Net power) Observe that the expression (20a) is a degenerate pairing, since $P^{\text {net }}=0$ for any $\left(f^{q}, f^{\phi}, f^{B L}, f^{B R}\right)$ does not imply that the $\left(e_{0}^{q}, e_{0}^{\phi}, e_{S}^{q}, e_{S}^{\phi}, e^{B L}, e^{B R}\right)$ is zero. On the other hand the expression (22) is a non-degenerate pairing.

Elimination of $f^{q}, f^{\phi}, e_{0}^{q}, e_{0}^{\phi}, e_{S}^{q}, e_{S}^{\phi}$ from (18),(21) gives

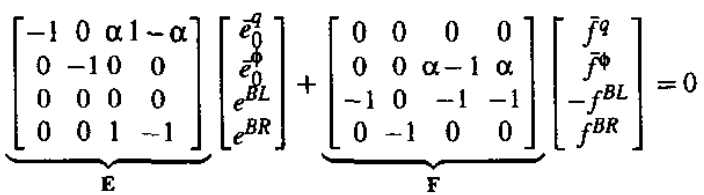

Equation (23) represents the spatially discretised interconnection structure of this cell expressed by means of the port variables. In the sequel we investigate the properties of (23). Denote with $\mathcal{D}$ the space of admissible efforts, $\mathbf{e}$, and the flows, $\mathbf{f}$, such that (23) is satisfied, i.e.

$$
\mathcal{D}=\left\{(\mathbf{f}, \mathbf{e}) \in \mathbb{R}^{8}: \mathbf{E e}+\mathbf{F f}=0\right\} .
$$

Proposition $2 \mathcal{D}$ is a Dirac structure with the respect to the bilinear form

$$
\ll\left(\mathbf{f}^{1}, \mathbf{e}^{1}\right),\left(\mathbf{f}^{2}, \mathbf{e}^{2}\right) \gg=\left(\mathbf{e}^{1}\right)^{\mathrm{T}} \mathbf{f}^{2}+\left(\mathbf{e}^{2}\right)^{\mathrm{T}} \mathbf{f}^{1} .
$$

Proof: $\mathcal{D}$ is a Dirac structure with respect to the bilinear form given by (25) if and only if the following two conditions are satisfied [1]: $\operatorname{rank}\left[\begin{array}{ll}\mathbf{E} & \mathbf{F}\end{array}\right]=4$, and $\mathbf{F E} \mathbf{E}^{\mathrm{T}}+\mathbf{E F}^{\mathrm{T}}=$ 0 . Straightforward computation shows that both conditions are satisfied.

\subsection{Discretization of the constitutive relations}

The flow variables $f^{q}(t, z)$ and $f^{\phi}(t, z)$ are approximated by (9). Since $f^{q}(t, z)$ and $f^{\phi}(t, z)$ are related to the energy variables $q(t, z)$ and $\phi(t, z)$, then $q(t, z)$ and $\phi(t, z)$ are approximated on $z$ by

$$
\begin{aligned}
& q(t, z)=Q(t) \cdot{ }^{1} b^{q}(z), \\
& \phi(t, z)=\Phi(t) \cdot{ }^{1} b^{\phi}(z),
\end{aligned}
$$

where

$$
\begin{aligned}
& \dot{Q}(t)=\bar{f}^{q}(t), \\
& \dot{\Phi}(t)=\bar{f}^{\phi}(t) .
\end{aligned}
$$

Observe that $Q(t)$ represents the total amount of charge of the cell and $\Phi(t)$ represents the total amount of flux of the cell.

The electric energy of the cell is given by

$$
H^{q}(t)=\frac{1}{2} \int_{Z} e^{q}(t, z) \wedge q(t, z)
$$

or equivalently as

$$
H^{q}(t)=\frac{1}{2} \int_{z} * \frac{1}{C(z)} q(t, z) \wedge q(t, z) .
$$

First, the electrical energy of the cell calculated by means of discretization of (28a) is

$$
\begin{aligned}
& H^{q}(Q(t))=\frac{1}{2} \int_{Z} e^{q}(t, z) \wedge q(t, z) \\
& =\frac{1}{2} \int_{z}\left(e_{0}^{q}(t) \cdot b_{0}^{q}(z)+e_{S}^{q}(t) \cdot b_{S}^{q}(z)\right) \wedge Q(t) \cdot{ }^{1} b^{q}(z) \\
& =\frac{1}{2}\left(\alpha e_{0}^{q}(t)+(1-\alpha) e_{S}^{q}(t)\right) Q(t)=\frac{1}{2} \vec{e}_{0}^{q}(t) Q(t)
\end{aligned}
$$

The electrical energy calculated by means of discretization of $(28 b)$ is

$$
H^{q}(t)=\frac{1}{2} \int_{Z} * \frac{Q(t)^{1} b^{q}(z)}{C(z)} \wedge Q(t)^{1} b^{q}(z)=\frac{Q^{2}(t)}{2 C},
$$

where

$$
C^{-1}=\int_{z} \frac{1}{C(z)} *^{1} b^{q}(z) \wedge^{1} b^{q}(z)
$$


Comparing the expressions for the energy yields

$$
\bar{e}^{q}(t)=\frac{Q(t)}{C}
$$

By using the same reasoning as before, one proves that

$$
\vec{e}^{\phi}(t)=\frac{\Phi(t)}{L}
$$

where

$$
L^{-1}=\int_{\mathbf{Z}} \frac{1}{L(z)} *^{1} b^{\phi}(z) \wedge^{1} b^{\phi}(z)
$$

Now it is clear that (29b) and (29c) can be rewritten as

$$
\begin{aligned}
& \tilde{e}^{q}=\frac{\partial H(Q, \Phi)}{\partial Q}=\frac{Q}{C}, \\
& \tilde{e}^{\phi}=\frac{\partial H(Q, \Phi)}{\partial \Phi}=\frac{\Phi}{L},
\end{aligned}
$$

where $H(Q, \Phi)$, the total energy of the cell, is given by

$$
H(Q, \Phi)=\frac{Q^{2}}{2 C}+\frac{\Phi^{2}}{2 L} .
$$

The equations (23),(interconnection structure) and (27), (29) (constitutive relations of the magnetic and electric ports) represent a finite dimensional model of the transmission line.

\subsection{Properties of the Finite-dimensional model}

(i) As noted before the interconnection structure of the finite dimensional model is a Dirac structure. Since the constitutive relations of the magnetic and electric ports obey Hamiltonian formalism then the finite dimensional model ((23), (27), (30)) represents a port Hamiltonian system!

(ii) Since the interconnection structure of the finite dimensional model is a Dirac structure its net power is zero. Consider $n$ cells of the transmission line, each interconnected to one another. On each cell a spatial approximation is performed and finite models derived. The cells are interconnected such that

$$
e_{i}^{B R}=e_{i+1}^{B L}, \quad f_{i}^{B R}=f_{i+1}^{B L}
$$

Then

$$
\begin{gathered}
0=P^{n e t}= \\
\sum_{i=0}^{n} \bar{f}_{i}^{q}(t) \vec{e}_{i}^{q}(t)+\sum_{i=0}^{n} \bar{f}_{i}^{\phi}(t) \vec{e}_{i}^{\phi}(t)-f^{B L}(t) e^{B L}(t) \\
+f^{B R}(t) e^{B R}(t) \\
\Rightarrow H(Q(t), \Phi(t))-f^{B}(t) e^{B L}(t)+f^{B R}(t) e^{B R}(t)=0
\end{gathered}
$$

where

$$
\begin{aligned}
& H(Q, \Phi)=\sum_{i=0}^{n} H_{i}\left(Q_{i}, \Phi_{i}\right) \\
& Q=\left[\begin{array}{lll}
Q_{0} & \cdots & Q_{n}
\end{array}\right]^{T}, \quad \Phi=\left[\begin{array}{lll}
\Phi_{0} & \cdots & \Phi_{n}
\end{array}\right]^{T}
\end{aligned}
$$

Relation (32) represents the power balance of the discretized model and it has the same form as the power balance of the distributed parameter system.

(iii) Inserting (27a), (21a) into (16) and taking into account the boundary conditions in ( 3 ) gives

$$
\dot{Q}_{i}(t)=f_{i}^{B L}(t)-f_{i}^{B R}(t)
$$

The boundary variables of the $i^{t h}$ and the $i+1^{t h}$ are related as follows:

$$
f_{0}^{B L}=f^{B L}, e_{0}^{B L}=e^{B L}, f_{n}^{B R}=f^{B R}, e_{n}^{B R}=e^{B R}
$$

Summation of relation (33) for $i=0 \ldots n$, where (34) is taken into account, gives

$$
\dot{Q}(t)=f^{B L}(t)-f^{B R}(t)
$$

where $Q=\sum_{i=0}^{n} Q_{i}$ represents the total charge of the transmission line. The previous relation represents a dynamic invariant and it has the same form as (6). Similarly, it can be proved that the second dynamic invariant is given by

$$
\Phi=e^{B L}-e^{B R}
$$

where $\Phi=\sum_{i=0}^{n} \Phi_{i}$ represents the total flux of the transmission line. Hence the dynamic invariants are preserved in the finite dimensional model.

(iv) The conservation of energy for the infinitedimensional port-Hamiltonian system was explained in Section 3.1. Due to the power-conservation property of the Dirac structure, defined by the vanishing of the bilinear form when restricted to $\mathcal{D}$, any distributed parameter port-Hamiltonian system satisfies along it's trajectories the energy balance [4]. In the finite dimensional model, the vanishing of the bilinear form when restricted to the finite-dimensional Dirac structure is indicated by (32). Hence, we have the energy conservation law for the finite-dimensional model also. Once we have the energy balance law, the property of passivity follows trivially, just as in Section 3.1, where of course the Hamiltonian remains positive-definite also after the spatial discretization. Hence for the finite-dimensional model

$$
\begin{aligned}
& H(Q(T), \Phi(T))-H\left(Q(0), \Phi(0)=\int_{0}^{T} s\left(e^{B}, f^{B}\right)\right. \\
& \Rightarrow \int_{0}^{T} s\left(e^{B}, f^{B}\right) \geq-H(Q(0), \Phi(0)
\end{aligned}
$$


where $s\left(e^{B}, f^{B}\right)$ is the supplied power just as in Section 3.1. (35) is the property of passivity, which implies that the input output maps of the finitedimensional system for every $(Q(0), \Phi(0)$ are passive.

\subsection{Discussion}

In (20b) we have defined the variable $\alpha$ which depends on the choice of the independent functions. Usual approximations of the Telegrapher's equations result in LC laddernetworks. The approximation that we propose are either LC ladder networks or mirrored-LC ladder networks (which implies that we can use either a voltage or a current source as the input) which include transformers. The results of the approximation then depend on the choice of independent functions. So if we choose $\alpha=0$, which corresponds to the independent functions being simply Dirac delta functions $\delta(z-0)$, then the approximation is the LC ladder network with accuracy equal to $\frac{1}{n}$, where $n$ is the number of cells. If we choose $\alpha=1$, which corresponds to the independent functions being $\delta(z-S)$, then the approximation is the mirrored-LC ladder network with an accuracy equal to $\frac{1}{n}$. However if we choose $\alpha=0.5$ for constant $C(z)$ and $L(z)$ then we obtain LC ladder networks with transformers, and with an accuracy equal to $\frac{1}{n^{2}}$. In the case that $C(z)$ and $L(z)$ are varying functions, then we can always choose independent functions such that the accuracy is equal to $\frac{1}{n^{2}}$. Note that the accuracy of the approximation can be improved by an appropriate choice of the independent functions. In fact as it turns out an optimal choice of $\alpha$ for constant $C(z)$ and $L(z)$ is 0.5 , and the accuracy is then $\frac{1}{n^{2}}$.

\subsection{Extension to higher dimensional cases}

We give a brief outline of the procedure for higher dimensional cases. Consider the two-dimensional wave equation. We can express the wave equation as a port-Hamiltonian system with the energy variables defined as the 2 -form infinitesimal kinetic momentum $\rho\left(t, z_{1}, z_{2}\right)$, the 1-form infinitesimal elastic strain and the co-energy variables defined as the 1 -form infinitesimal stress and the 0 -form velocity. The co-energy variables are related to the energy variables by the constitutive relations of the wave equation. For the approximation we choose square grids. The approximation of the 0 -forms and the 1 -forms of the wave equation is done as in equations (9) and (11). We define $f^{\rho}\left(t, z_{1}, z_{2}\right)=$ $\frac{\partial p\left(t, z_{1}, z_{2}\right)}{\partial t}$. The 2 -form infinitesimal rate kinetic momentum is approximated as $f^{\rho}\left(t, z_{1}, z_{1}\right)=f^{\rho}(t)^{2} b^{\rho}\left(z_{1}, z_{2}\right)$ where we make the assumption that ${ }^{2} b^{\rho}\left(z_{1}, z_{2}\right)$ satisfies $\int^{2} b^{\rho}\left(z_{1}, z_{2}\right)=$ 1 with the integral defined on the square grid. This assumption is physically motivated since the infinitesimal kinetic momentum is approximated as $\rho\left(t, z_{1}, z_{2}\right)=\rho(t) \cdot{ }^{2} b^{\rho}\left(z_{1}, z_{2}\right)$ and since we choose ${ }^{2} b^{\rho}\left(z_{1}, z_{2}\right)$ to satisfy the above assumption, hence $\rho(t)$ represents the total kinetic momentum on the approximating square grid. Then we proceed as done in Section (3.1) and derive the spatially discretized interconnection structure. And the discretization of the constitutive relations follow the same reasoning as in Section (3.2). The extension to port-Hamiltonian systems over 3-dimensional spatial domain is then fairly obvious. We approximate an infinitesimal 3-form with a function which satisfies the condition that it's integral over a cube grid is equal to 1 . And we proceed as before.

\section{Conclusions}

Numerical methods for the solutions of PDE's usually assume that the boundary conditions are given. In the framework's of network modeling and control, the boundary conditions represent the interaction of the various components of the system with each other and with the environment. Hence the need to approximate distributed parameter systems while retaining the energetic port-structure. We adopt a point of view introduced by Bossavit, [6], and solve the general problem of approximation including free boundary conditions, for the telegrapher's equations. Indeed, this procedure can be adopted for other types of 1-dimensional systems which can be modeled as port-Hamiltonian systems, like the 1-dimensional compressible fluid, the vibrating string, the Timoshenko beam etc. Also, approximating distributed parameter systems with dissipation can be easily incorporated into this procedure. Also important in such approximations are to preserve the physical properties of the original system. In this paper we have shown how the portHamiltonian structure can be preserved, as well as some other physical properties. The extension of our procedure to higher-dimensional cases is conceptually straightforward.

\section{References}

[1] A.J. van der Schaft and B.M. Maschke. The hamiltonian formulation of energy conserving physical systems with externai ports. Archiv fur Electronik und Ubertragungstechnik, pp. 362-37I, 49:362-371, 1995.

[2] B.M. Maschke, A.J. van der Schaft, and P.C. Breedveld. An intrinsic hamiltonian formulation of network dynamics: Non-standard poisson structures and gyrators. Journal of Franklin Institute, 329(5), pages 923-966, 1992.

[3] A.J. van der Schaft. $L_{2}$-Gain and Passivity Techniques in Nonlinear Control. Springer-Verlag, 2000.

[4] A.J. van der Schaft and B.M. Maschke. Hamiltonian formulation of distributed-parameter systems with boundary energy flow. Journal of Geometry and Physics, to appear, 2002.

[5] P.J. Olver. Applications of Lie groups to differential equations. Springer-Verlag, 1993.

[6] A. Bossavit. Differential forms and the computation of fileds and forces in electromagnetism. European Journal of Mechanics, B/Fluids, 10(5):474-488, 1991.

[7] G. Golo, V. Talasila, A.J. van der Schaft, and B.M. Maschke. Hamiltonian discretizations of the telegrapher's equations. In Preparation, 2002. 